\title{
Scattering in a 2-D Optical Waveguide
}

\author{
Rolando Magnanini * Fadil Santosa ${ }^{\dagger}$
}

\begin{abstract}
We consider the problem of scattering in a planar optical waveguide. An incident wave, in the form of a guided mode, is sent along the waveguide. It encounters an inhomogeneity in the core region of the waveguide, and is scattered. We use the Green's function for the planar waveguide to derive a Lippman-Schwinger equation. We show that the integral equation admits a unique solution. The scattering problem is solved under the Born approximation in several numerical examples.
\end{abstract}

\section{Introduction}

Our work is motivated by the need to obtain a detailed description of wave phenomena in optical waveguides. This type of information is important in engineering design of optical communication devices. Although the field of optical waveguide is quite mature, there are areas where more investigation is warranted. The problem under consideration is an example.

An optical waveguide is referred to as inhomogeneous if the index of refraction depends on the direction of propagation of a guided wave. To be precise, let the waveguide occupy all of $(x, z) \in \mathbb{R}^{2}$. The region $|x|<h$ is considered the core of the fibre, while the remainder is considered the cladding. The index of refraction in the core is larger than that in the cladding. Therefore, a homogeneous, or unperturbed, waveguide has index of refraction given by

$$
n_{0}(x)=\left\{\begin{array}{lll}
n_{\mathrm{cl}} & \text { for } & |x|>h \\
n_{\mathrm{co}} & \text { for } & |x|<h
\end{array} .\right.
$$

* Dipartimento di Matematica U. Dini, University of Firenze, viale Morgagni 67/A, 50134 Firenze, Italy

${ }^{\dagger}$ School of Mathematics, University of Minnesota, Vincent Hall, 206 Church Street S.E., Minneapolis, MN 55455, USA 
Here we assumed that both $n_{\mathrm{cl}}$ and $n_{\mathrm{co}}$ are constants for simplicity. Indeed, we expect our method to work also for the case where $n_{\text {co }}$ is a function of $x$, which is more realistic. The perturbed waveguide has an index of refraction which depend on $x$ and $z$, and we write it as

$$
n(x, z)^{2}=n_{0}(x)^{2}+m(x, z),
$$

where $m(x, z)$ is supported in $\Omega:=[-a, a] \times[-b, b]$. For simplicity, we assume that $a \leq h$. Note that we define the perturbation $m(x, z)$ through the square of the index of refraction for convenience.

The perturbation in the index of refraction can be caused by an imperfection. It can also be introduced as part of a design, such as in fiber Bragg grating.

The problem we wish to solve is to find $u(x, z)$ satisfying Helmholtz's equation

$$
\Delta u+k^{2} n(x, z)^{2} u=0 \quad(x, z) \in \mathbb{R}^{2} .
$$

If the incident field is given, we can decompose $u(x, z)$ as

$$
u(x, z)=u_{\text {inc }}(x, z)+u_{\text {scat }}(x, z) .
$$

The scattered field $u_{\text {scat }}$ must satisfy some form of radiation condition. Unfortunately, not much is known about the exact form of this condition due to the fact that the waveguide extends from $-\infty$ to $\infty$ in $x$. The work by Nosich and Shestopalov [6] discusses the issue of boundary conditions.

We avoid this issue by first obtaining a representation for the Green's function of the homogeneous waveguide. The Green's function we obtain is based on the requirement that waves be outgoing (in the sense to be made precise) and remain bounded.

Using the obtained Green's function, we derive a Lippman-Schwinger integral equation for the solution of the inhomogeneous waveguide problem. We will attempt to make connection of our results with those from couple-mode theory $[7,4,5]$. We show that under rather general conditions, the integral equation admits a unique solution. We end the paper with numerical results which are based on Born approximations. This approximation is valid in many applications because the perturbation in the index of refraction is often quite small. 


\section{Green's function for unperturbed waveguide}

We summarize and specialize the results obtained in [3]. The Helmholtz equation in the unperturbed waveguide is

$$
\Delta u+k^{2} n_{0}(x)^{2} u=0 \quad(x, z) \in \mathbb{R}^{2},
$$

where $n_{0}(x)$ is given in (1). We note that if the solution is of the form

$$
u(x, z)=v(x, \beta) \exp i k \beta z,
$$

then $v(x, \beta)$ satisfies

$$
v^{\prime \prime}+k^{2}\left[n(x)^{2}-\beta^{2}\right] v=0 .
$$

We rewrite the last equation by introducing

$$
q(x)=\left\{\begin{array}{lll}
0 & \text { for } & |x|<h \\
k^{2}\left(n_{\mathrm{co}}^{2}-n_{\mathrm{cl}}^{2}\right) & \text { for } \quad|x|>h
\end{array},\right.
$$

and $d^{2}=k^{2}\left(n_{\mathrm{co}}^{2}-n_{\mathrm{cl}}^{2}\right)$. Letting $\lambda=k^{2}\left(n_{\mathrm{co}}^{2}-\beta^{2}\right)$, we get

$$
v^{\prime \prime}+(\lambda-q) v=0 \quad \text { in } \quad \mathbb{R} .
$$

We view $\lambda$ as the 'eigenvalue' parameter.

We construct two linearly independent solutions $v_{\mathrm{a}}(x, \lambda)$ and $v_{\mathrm{S}}(x, \lambda)$; the subscripts indicate the symmetry of the solution with respect to the $x$ axis. Let $Q=\sqrt{\lambda-d^{2}}$, then the solutions are given by

$v_{\mathbf{S}}(x, \lambda)= \begin{cases}\cos (h \sqrt{\lambda}) \cos Q(x-h)-\frac{\sqrt{\lambda}}{Q} \sin (h \sqrt{\lambda}) \sin Q(x-h), & \text { if } x \geq h, \\ \cos (x \sqrt{\lambda}), & \text { if }|x|<h, \\ \cos (h \sqrt{\lambda}) \cos Q(x+h)+\frac{\sqrt{\lambda}}{Q} \sin (h \sqrt{\lambda}) \sin Q(x+h), & \text { if } x \leq-h,\end{cases}$

and

$v_{\mathrm{a}}(x, \lambda)= \begin{cases}\sin (h \sqrt{\lambda}) \cos Q(x-h)+\frac{\sqrt{\lambda}}{Q} \cos (h \sqrt{\lambda}) \sin Q(x-h), & \text { if } x \geq h, \\ \sin (x \sqrt{\lambda}), & \text { if }|x|<h, \\ -\sin (h \sqrt{\lambda}) \cos Q(x+h)+\frac{\sqrt{\lambda}}{Q} \cos (h \sqrt{\lambda}) \sin Q(x+h), & \text { if } x \leq-h .\end{cases}$

Bounded solutions $v(x, \lambda)$ exist for certain values of $\lambda$ : 
1. A discrete set of $\lambda$ for $0<\lambda<d^{2}$ satisfying

$$
\begin{array}{lll}
\sqrt{d^{2}-\lambda}-\sqrt{\lambda} \tan (h \sqrt{\lambda})=0, & \text { for } & v_{\mathrm{S}}(x, \lambda), \\
\sqrt{d^{2}-\lambda}+\sqrt{\lambda} \cot (h \sqrt{\lambda})=0, & \text { for } & v_{\mathrm{a}}(x, \lambda) .
\end{array}
$$

These are associated with the guided modes. Let us say that we have $m_{\mathbf{S}}$ and $m_{\mathrm{a}}$ roots to the above equations, respectively.

2. For $d^{2}<\lambda<k^{2} n_{\text {co }}^{2}$ where the solutions are associated with the radiation modes.

3. For $\lambda>k^{2} n_{\text {co }}^{2}$, making $\beta$ imaginary, and the solutions are associated with the evanescent modes.

What we show is that the solutions $v_{\mathrm{s}}(x, \lambda)$ and $v_{\mathrm{a}}(x, \lambda)$ can be viewed as transform kernels, with the corresponding set of $\lambda$ for each solution as the transform variable. The transform of a function $f(x)$ is

$$
F^{j}(\lambda)=\int_{-\infty}^{+\infty} f(x) v_{j}(x, \lambda) d x
$$

The inverse transform is given by

$$
f(x)=\sum_{j \in\{\mathbf{s}, \mathbf{a}\}} \int F^{j}(\lambda) d \rho^{j}(\lambda),
$$

where the weight

$$
\begin{aligned}
\left\langle d \rho^{\mathrm{s}}, \eta\right\rangle= & \sum_{l=1}^{m_{\mathrm{s}}} \frac{\sqrt{d^{2}-\lambda_{l}^{\mathrm{s}}}}{1+h \sqrt{d^{2}-\lambda_{l}^{\mathrm{s}}}} \eta\left(\lambda_{l}^{\mathrm{S}}\right)+ \\
& \frac{1}{2 \pi} \int_{d^{2}}^{+\infty} \frac{\sqrt{\lambda-d^{2}}}{\left(\lambda-d^{2}\right) \cos ^{2}(h \sqrt{\lambda})+\lambda \sin ^{2}(h \sqrt{\lambda})} \eta(\lambda) d \lambda, \\
\left\langle d \rho^{\mathrm{a}}, \eta\right\rangle= & \sum_{l=1}^{m_{\mathrm{a}}} \frac{\sqrt{d^{2}-\lambda_{l}^{\mathrm{a}}}}{1+h \sqrt{d^{2}-\lambda_{l}^{\mathrm{a}}}} \eta\left(\lambda_{l}^{\mathrm{a}}\right)+ \\
& \frac{1}{2 \pi} \int_{d^{2}}^{+\infty} \frac{\sqrt{\lambda-d^{2}}}{\left(\lambda-d^{2}\right) \sin ^{2}(h \sqrt{\lambda})+\lambda \cos ^{2}(h \sqrt{\lambda})} \eta(\lambda) d \lambda .
\end{aligned}
$$


The method employed in arriving at these weights is explained in generality in $[8,1]$; the details can be found in [3].

The desired Green's function, $g(x, z ; \xi, \zeta)$, satisfies

$$
\Delta g+k^{2} n_{0}(x)^{2} g=\delta(x-\xi) \delta(z-\zeta) .
$$

By applying the transform method above, we arrive at a representation for $g(x, z ; \xi, \zeta)$

$$
g(x, z, \xi, \zeta)=\sum_{j \in\{\mathbf{s}, \mathbf{a}\}} \int v_{j}(x, \lambda) v_{j}(\xi, \lambda) \frac{e^{i k \beta|z-\zeta|}}{2 i k \beta} d \rho^{j}(\lambda) .
$$

Recall that $\lambda=k^{2}\left(n_{\text {co }}^{2}-\beta^{2}\right)$. The Green's function has been constructed so that all the waves are outgoing. As mentioned earlier, we do not know the correct form of the outgoing boundary condition for $g(x, z ; \xi, \zeta)$. But the transform method allows us to identify incoming and outgoing components of the transform of $g$. The reader is referred to [3] for numerical examples of the Green's function.

\section{Lippman-Schwinger equation}

We return to finding $u(x, z)$ satisfying (3). Suppose we know the incident wave is $u_{\text {inc }}(x, z)$. Using the decomposition $u=u_{\text {inc }}+u_{\text {scat }}$ and (2), we find that $u_{\text {scat }}(x, z)$ satisfies

$$
\Delta u_{\text {scat }}+k^{2} n_{0}(x) u_{\text {scat }}=-k^{2} m(x, z) u .
$$

Since we have the Green's function, and $m(x, z)$ is supported in $\Omega=[-a, a] \times$ $[-b, b]$, we can write the solution to (13) as

$$
u_{\text {scat }}(x, z)=-k^{2} \int_{\Omega} m(\xi, \zeta) u(\xi, \zeta) g(x, z ; \xi, \zeta) d \xi d \zeta .
$$

The field $u(x, z)$ in $\Omega$ is unknown and needs to be determined by solving the integral equation

$$
u(x, z)=u_{\text {inc }}(x, z)-k^{2} \int_{\Omega} m(\xi, \zeta) u(\xi, \zeta) g(x, z ; \xi, \zeta) d \xi d \zeta .
$$

Once this is determined, we can use (14) to find the scattered waves. 
To show that (15) has a solution, we use the approach presented in [2]. Define the operator $T$ through

$$
T u(x, z):=-k^{2} \int_{\Omega} m(\xi, \zeta) u(\xi, \zeta) g(x, z ; \xi, \zeta) d \xi d \zeta \text { for }(x, z) \in \Omega .
$$

We form the Neumann series

$$
u(x, z)=\sum_{l=0}^{\infty} u_{l}(x, z)
$$

with

$$
u_{0}(x, z)=u_{\text {inc }}(x, z), \quad u_{n}(x, z)=T u_{n-1}(x, z) .
$$

We need to show that the series converges. To this end, we need to establish that $\|T\|$ is bounded. From (16), we see that

$$
|T u(x, z)| \leq k^{2} M|| u \|_{\infty} \int_{\Omega}|g(x, z ; \xi, \zeta)| d \xi d \zeta .
$$

Here $M=\sup _{\Omega} m(x, z)<\infty$. Let $K:=k^{2} M \int_{\Omega}|g(x, z ; \xi, \zeta)| d \xi d \zeta$. Then as long $K<1$, the Neumann series converges. What remains is to show that $\int_{\Omega}|g(x, z ; \xi, \zeta)| d \xi d \zeta$ is bounded.

Consider the $x$-symmetric contribution to the Green's function. We use the weight in (10) and the representation for the Green's function in (12) to write

$$
\begin{gathered}
g_{\mathrm{S}}(x, z ; \xi, \zeta)=\sum_{l=1}^{m_{\mathrm{S}}} v_{\mathrm{S}}\left(x, \lambda_{l}\right) v_{\mathrm{S}}\left(\xi, \lambda_{l}\right) \frac{\exp i k \beta_{l}|z-\zeta|}{2 i k \beta_{l}} \frac{\sqrt{d^{2}-\lambda_{l}}}{1+h \sqrt{d^{2}-\lambda_{l}}} \\
\quad+\frac{1}{2 \pi} \int_{d^{2}}^{\infty} v_{\mathbf{S}}(x, \lambda) v_{\mathbf{S}}(\xi, \lambda) \frac{\exp i k \beta|z-\zeta|}{2 i k \beta} \frac{\sqrt{\lambda-d^{2}}}{\left(\lambda-d^{2}\right) \cos ^{2}(h \sqrt{\lambda})+\lambda \sin ^{2}(h \sqrt{\lambda})} d \lambda .
\end{gathered}
$$

For $|x|<a$ we have from (6)

$$
\left|v_{\mathrm{S}}\left(x, \lambda_{l}\right)\right| \leq 1, \quad l=1, \cdots, m_{\mathrm{S}},
$$

and moreover, it is easy to show that

$$
\left|v_{\mathbf{S}}(x, \lambda)\right| \leq \sqrt{\frac{\left(\lambda-d^{2}\right) \cos ^{2}(h \sqrt{\lambda})+\lambda \sin ^{2}(h \sqrt{\lambda})}{\lambda-d^{2}}}, \quad \lambda>d^{2} .
$$


We have then, for $(x, z)$ and $(\xi, \zeta)$ in $\Omega$

$$
\begin{aligned}
& \left|g_{\mathrm{S}}(x, z ; \xi, \zeta)\right| \leq \sum_{l=1}^{m_{\mathrm{S}}} \frac{1}{2 k \beta_{l}} \frac{\sqrt{d^{2}-\lambda_{l}}}{1+h \sqrt{d^{2}-\lambda_{l}}} \\
& \quad+\frac{1}{2 \pi} \int_{d^{2}}^{k^{2} n_{\mathrm{co}}^{2}} \frac{1}{2 k \beta} \frac{d \lambda}{\sqrt{\lambda-d^{2}}}+\frac{1}{2 \pi} \int_{k^{2} n_{\mathrm{co}}^{2}}^{\infty} \frac{e^{-k|\beta||z-\zeta|}}{2 k|\beta|} \frac{d \lambda}{\sqrt{\lambda-d^{2}}} .
\end{aligned}
$$

We observe now that $\beta_{l} \geq n_{\mathrm{cl}}, l=1, \cdots, m_{\mathrm{s}}$ and apply the substitutions $\lambda=$ $d^{2}+\mu^{2}$ and $\lambda=k^{2} n_{\text {co }}+\nu^{2}$ to the two integrals to obtain

$$
\begin{aligned}
\left|g_{\mathrm{s}}(x, z ; \xi, \zeta)\right| \leq & \frac{m_{\mathrm{s}}}{2 h k n_{\mathrm{cl}}}+\frac{1}{2 \pi} \int_{0}^{k n_{\mathrm{cl}}} \frac{d \mu}{\sqrt{k^{2} n_{\mathrm{cl}}^{2}-\mu^{2}}}+\frac{1}{2 \pi} \int_{0}^{\infty} \frac{e^{-\nu|z-\zeta|}}{\sqrt{k^{2} n_{\mathrm{cl}}^{2}+\nu^{2}}} d \nu \\
\leq & \frac{m_{\mathrm{s}}}{2 h k n_{\mathrm{cl}}}+\frac{1}{4}+\frac{1}{2 \pi} \int_{0}^{k n_{\mathrm{co}}} \frac{d \nu}{\sqrt{k^{2} n_{\mathrm{cl}}^{2}+\nu^{2}}} \\
& +\frac{1}{2 \pi} \int_{k n_{\mathrm{co}}}^{\infty} \frac{e^{-\nu|z-\zeta|}}{\sqrt{k^{2} n_{\mathrm{cl}}^{2}+\nu^{2}}} d \nu \\
\leq & \frac{m_{\mathrm{S}}}{2 h k n_{\mathrm{cl}}}+\frac{1}{4}+\frac{1}{2 \pi} \log \left(\frac{n_{\mathrm{co}}+\sqrt{n_{\mathrm{co}}^{2}+n_{\mathrm{cl}}^{2}}}{n_{\mathrm{cl}}}\right) \\
& +\frac{1}{2 \pi} \int_{k n_{\mathrm{co}}}^{\infty} \frac{e^{-\nu|z-\zeta|}}{\sqrt{k^{2} n_{\mathrm{cl}}^{2}+\nu^{2}}} d \nu .
\end{aligned}
$$

From the equation satisfied by $\lambda_{l}$ 's, we know that $m_{\mathrm{S}} \leq 1+\frac{h d}{\pi}$. Finally, integrating the latter over $\Omega$ and interchanging the order of integration, we get

$$
\begin{aligned}
\int_{\Omega}\left|g_{\mathrm{s}}(x, z ; \xi, \zeta)\right| d \xi d \zeta \leq a b\left[1+\frac{2(\pi+h d)}{\pi h k n_{\mathrm{cl}}}+\frac{2}{\pi} \log \left(\frac{4 n_{\mathrm{co}}}{n_{\mathrm{cl}}}\right)\right] \\
+\frac{a}{\pi} \int_{k n_{\mathrm{co}}}^{\infty} \frac{d \nu}{\nu \sqrt{k^{2} n_{\mathrm{cl}}^{2}+\nu^{2}}} \\
\leq a b\left[1+\frac{2(\pi+h d)}{\pi h k n_{\mathrm{cl}}}+\frac{2}{\pi} \log \left(\frac{4 n_{\mathrm{co}}}{n_{\mathrm{cl}}}\right)\right] \\
+\frac{a}{\pi k n_{\mathrm{cl}}} \operatorname{arctanh}\left(\frac{n_{\mathrm{cl}}}{\sqrt{n_{\mathrm{co}}^{2}+n_{\mathrm{cl}}^{2}}}\right) .
\end{aligned}
$$




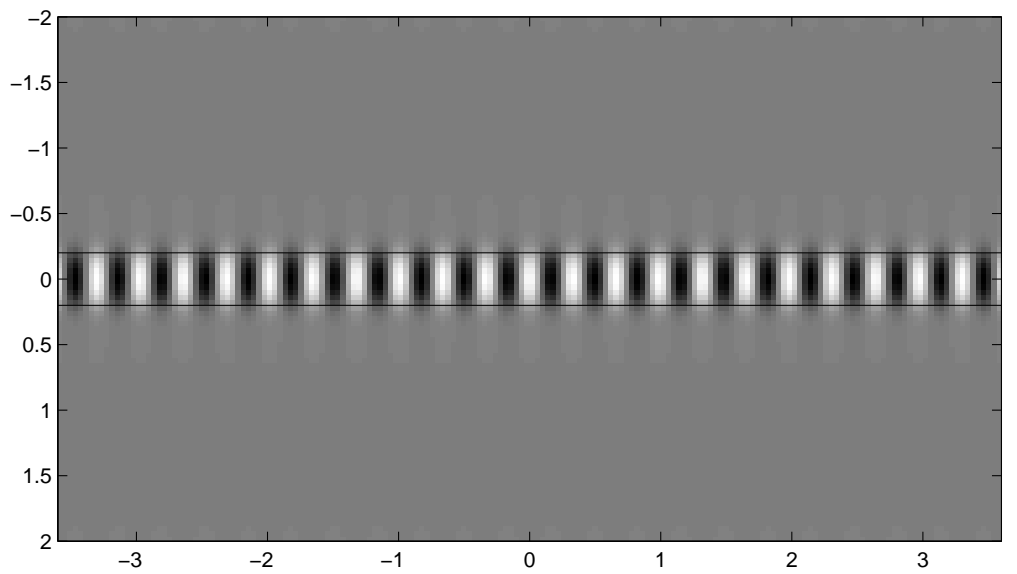

Figure 1: The real part of the incident field. The boundaries between the core and the cladding are highlighted with lines.

It is clear now that for every choice of $M$ we can choose $k$ sufficiently small so that in (17), $K<1$. Under these conditions, the Neumann series converges.

\section{Born approximation}

Under the Born approximation, we use the first term of the Neumann series. That is, we replace the $u(\xi, \zeta)$ with $u_{\text {inc }}(\xi, \zeta)$ in (15) to get

$$
u_{\text {scat }}(x, z) \approx-k^{2} \int_{\Omega} m(\xi, \zeta) u_{\text {inc }}(\xi, \zeta) g(x, z ; \xi, \zeta) d \xi d \zeta .
$$

This approximation will be accurate for small $k$ and $m(x, z)$.

We now connect this expression to modal theory from the study of optical waveguides. In modal theory, we consider solutions of the form

$$
u(x, z)=A v_{j}(x, \lambda) e^{i k \beta z},
$$

where $v_{j}(x, \lambda)$ have been given in (6) and (7). A mode is such a solution for a particular value of $\beta$. Recall that there are three types of modes depending 
on $\beta$ : guided, radiation, and evanescent. The inhomogeneity in the index of refraction couples an incoming incident mode with other modes. To see this, we use the representation for the Green's function (12), and insert it in (18). We get

$$
\begin{aligned}
u_{\text {scat }}(x, z) \approx & -k^{2} \int_{\Omega} m(\xi, \zeta) u_{\text {inc }}(\xi, \zeta) \\
& {\left[\sum_{j \in\{\mathrm{s}, \mathrm{a}\}} \int v_{j}(x, \lambda) v_{j}(\xi, \lambda) \frac{e^{i k \beta|z-\zeta|}}{2 i k \beta} d \rho^{j}(\lambda)\right] d \xi d \zeta . }
\end{aligned}
$$

Exchanging the order of integration and using the transform defined by the pair (8) and (9), we arrive at

$$
U_{\text {scat }}(z, \lambda) \approx-k^{2} \int_{\Omega} m(\xi, \zeta) u_{\text {inc }}(\xi, \zeta) \frac{e^{i k \beta|z-\zeta|}}{2 i k \beta} d \xi d \zeta .
$$

The above relation provides a formula to compute the coefficients of the scattered modes which are excited by the incident field. The individual scattered modes are of the form

$$
U_{\text {scat }}(z, \lambda) v_{j}(x, \lambda) \text {. }
$$

Particular care should be used in computing the excited guided modes as the eigenvalue $\lambda$ will be associated with either symmetric or antisymmetric $v_{j}(x, \lambda)$ (see (6) and (7)).

When the incident field is a mode, then

$$
u_{\text {inc }}(x, z)=A_{0} v_{j}\left(x, \lambda_{0}\right) e^{i k \beta_{0} z}, \quad \text { for } \quad j \in\{\mathrm{s}, \mathrm{a}\},
$$

where $\lambda_{0}=\sqrt{k^{2} n_{\text {co }}^{2}-\beta^{2}}$. The coefficients for the scattered modes are

$$
U_{\text {scat }}(z, \lambda) \approx-k^{2} A_{0} \int_{-b}^{b}\left[\int_{-a}^{a} m(\xi, \zeta) v_{j}\left(\xi, \lambda_{0}\right) d \xi\right] \frac{e^{i k \beta_{0} \zeta} e^{i k \beta|z-\zeta|}}{2 i k \beta} d \zeta .
$$

The formula above can be found in standard optical waveguide texts such as Snyder and Love [7] and Marcuse [4, 5].

To end this section, we describe some numerical results obtained using the Born approximation. In all the calculations, we used $k=10$. The homogeneous waveguide has $n_{\mathrm{cl}}=1$ and $n_{\mathrm{co}}=2$, with $h=0.2$. There are three guided modes; two symmetric and one antisymmetric. The incident wave in all the calculations is the lowest eigenvalue symmetric guided mode. The real part of the incident wave is shown in Figure 1. 
Three perturbation profiles are chosen. In the first one, the perturbation is a constant $m(x, z)$ supported on $[-0.5,0.5] \times[-.2, .2]$. The scattered wave, shown in Figure 2b, consists mostly of transmitted wave, some reflected wave, both of the same mode as the incident wave, and some radiation.

The second profile is a periodic perturbation whose period is half of the incident wavelength (in the $z$-direction). The function $m(x, z)$ is piecewise constant, and is zero for $|z|>h$. The periodicity is at the Bragg condition and is one that will couple the incident mode to forward and backward going modes of the same wavenumber. This phenomenon can be seen in Figure 3 (bottom). In comparing the scattered waves to that in Figure 2 (bottom), we see that there is a lot more reflected mode whose wavenumber is the same as the incident wave.

The final profile is an asymmetric constant perturbation as shown in Figure 4 (top). The resulting scattered wave includes symmetric and antisymmetric guided modes, in addition to radiation modes.

\section{Discussion}

We consider the problem of determining the scattered field in a 2-D optical waveguide when the guide contains an anomaly in the index of refraction. Starting with an explicit representation for the Green's function for a 2-D optical waveguide, we derived a Lippman-Schwinger integral equation for the solution of the scattering problem. We show that the integral equation admits a so-

lution. For the case where the index of refraction perturbation is small, we can approximate the scattered field using the Born approximation. The Born approximation is related to the classical modal analysis. The use of the Born approximation is demonstrated in several numerical computations. Finally, we note that while our results are for piecewise constant waveguides, we are confident that they can be extended to the case where the index of refraction in the core is variable. 

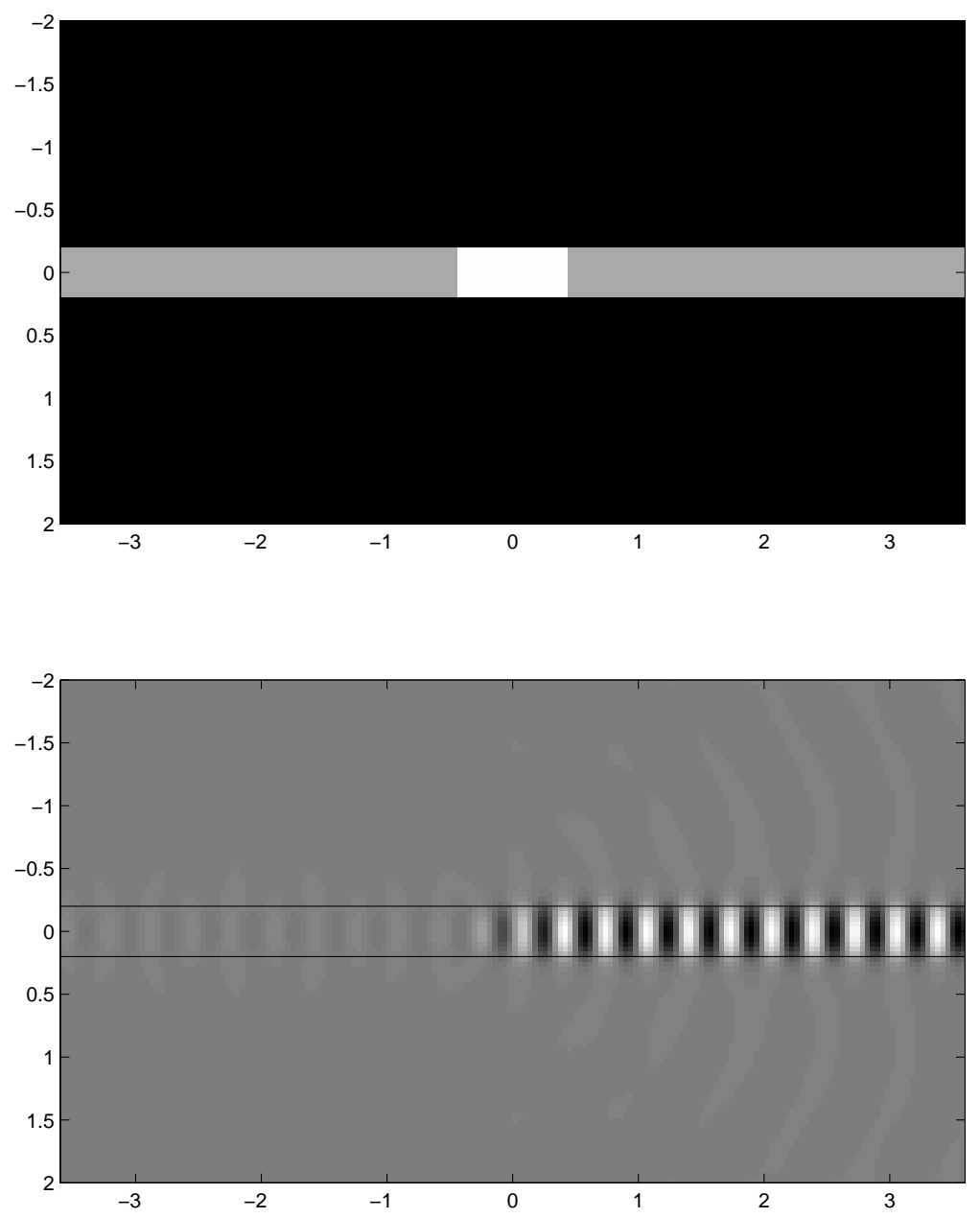

Figure 2: The block perturbation is supported on $[-0.5,0.5] \times[-.2, .2]$. The scattered field consists mostly of transmitted waves of the same form as the incident wave. However, a small amount of radiation has been generated. 

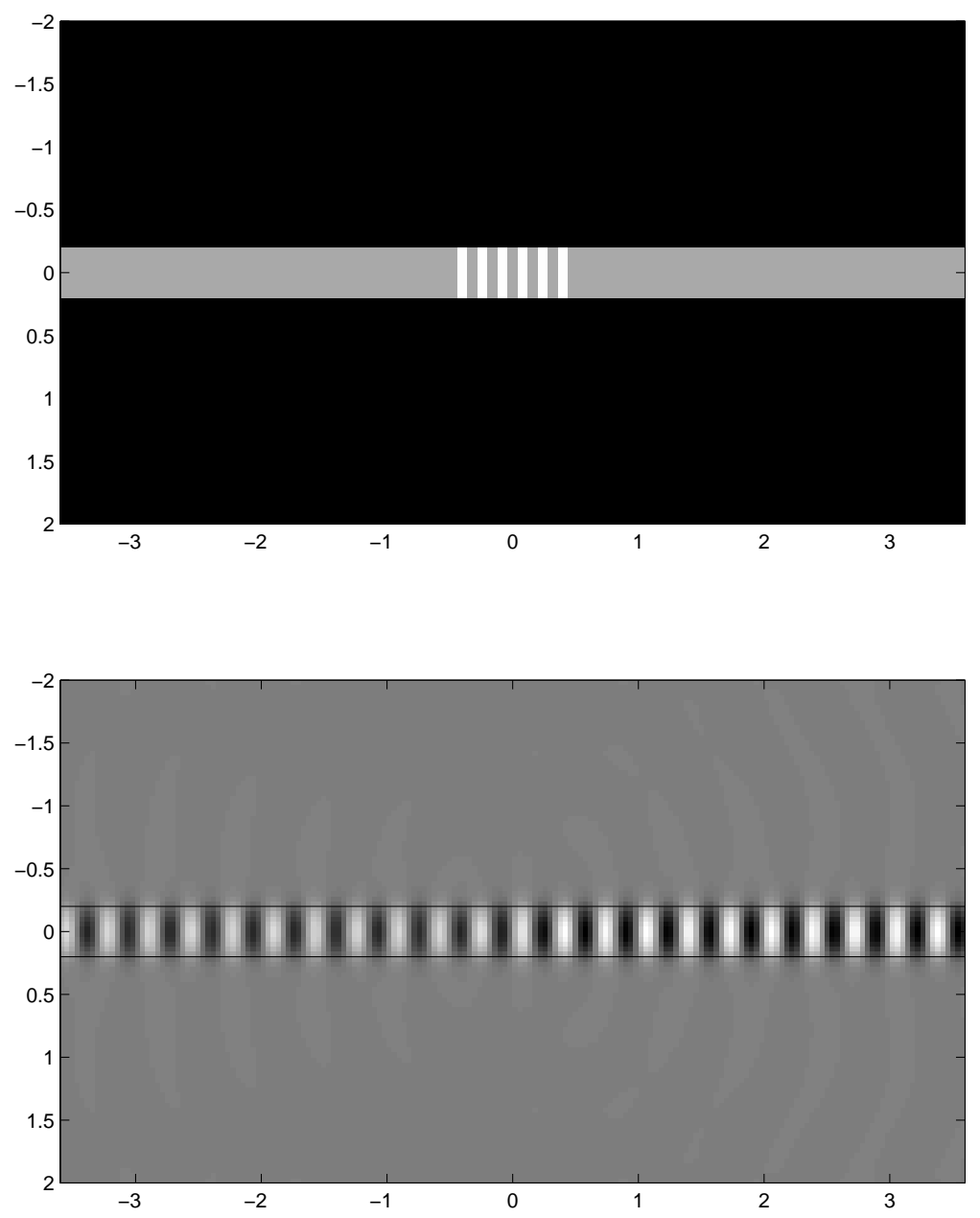

Figure 3: The perturbation, supported on $[-0.5,0.5] \times[-.2, .2]$, is piecewise constant and periodic at the Bragg condition. The scattered field has a strong reflection and transmission which are forward and backward going versions of the incident mode. The amount of radiation appears smaller than in Figure 2. 

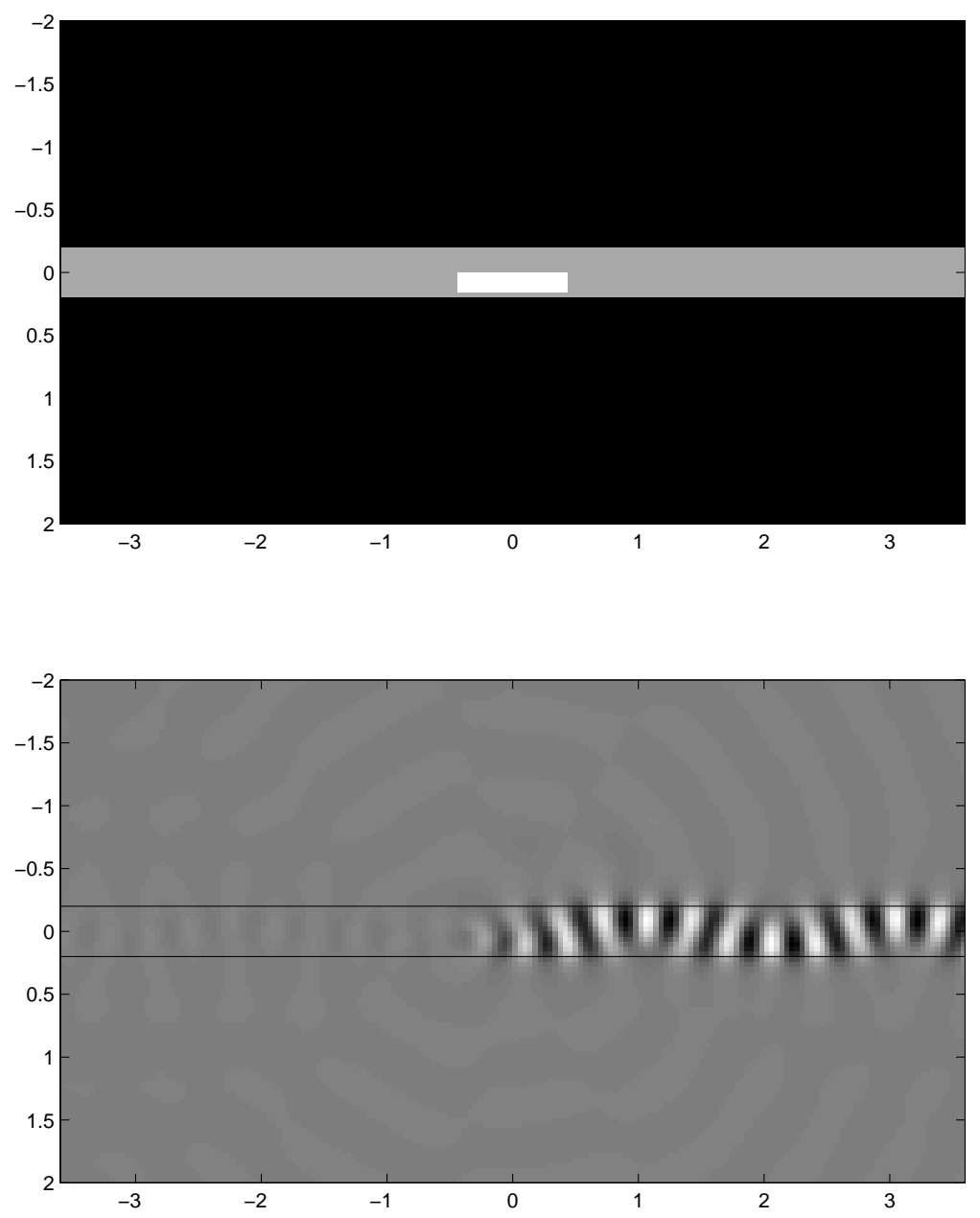

Figure 4: The perturbation is a small block and does not have symmetry in the vertical direction. Therefore, guided modes which are symmetric and antisymmetric are excited, in addition to radiated modes. 


\section{A dedication by F. S.}

Ralph Kleinman was a good friend, a valuable colleague, and a wonderful fatherin-law. In all these capacities, I have benefited greatly from my association with him. I often turned to him for advice and support. He always had time for me when I had technical questions about scattering or PDE. We shared many wonderful conversations and enjoyed many meals together. I will especially miss the times we spent together as a family. This paper is a small tribute to his memory.

\section{Acknowledgment}

The research of R. Magnanini is supported in part by CNR, Italy; the research of F. Santosa is supported in part by AFOSR under a MURI grant to the University of Delaware, and NSF.

\section{References}

[1] E.A. Coddington and N. Levinson, Theory of Ordinary Differential Equations, R.E. Krieger Publishing Company, Malabar (Florida) 1984.

[2] D. Colton and R. Kress, Inverse Acoustic and Electromagnetic Scattering Theory, 2nd Edition, Springer-Verlag, New York, 1998.

[3] R. Magnanini and F. Santosa, Wave propagation in a 2-D optical waveguide, submitted (1999).

[4] D. Marcuse, Theory of Dielectric Optical Waveguides, Academic Press, New York, 1974.

[5] D. Marcuse, Light Transmission Optics, Van Nostrand, New York, 1982.

[6] A.I. Nosich and V.P. Shestopalov, Radiation conditions and uniqueness theorems for open waveguides, Soviet J. Comm. Tech. Electron., 34 (1989), pp. 107-115.

[7] A.W. Snyder and D. Love, Optical Waveguide Theory, Chapman and Hall, London, 1983.

[8] E.C. Titchmarsh, Eigenfunction Expansions, 2nd Edition, Oxford at the Clarendon Press, Oxford, 1962. 